\title{
Método para determinar la separación entre barras y la curva de selección teórica de sistemas de grilla selectiva en redes de arrastre para peces
}

\author{
Mauricio Gálvez Larach \\ Departamento de Pesquerías \\ Instituto de Investigación Pesquera VIII Región S.A. \\ Casilla 350, Talcahuano, Chile \\ E-mail: inpesca@arauco.reuna.cl
}

Recibido 23 julio 1998; versión corregida 6 mayo 1999; aceptado 13 mayo 1999.

RESUMEN. En el artículo se propone un procedimiento numérico, basado en simulaciones bootstrap, para determinar en forma teórica la curva de selección a la talla de sistemas de grilla selectiva (SGS) en redes de arrastre para peces. Además, se propone una forma para determinar la separación inter-barra del dispositivo, basado en la estructura de tallas de las capturas obtenidas sin el dispositivo. El procedimiento considera que el ancho máximo $(A)$ del pez determina si éste escapa o no, además, no considera ninguna distribución particular para el ancho máximo de un pez de longitud $l$. La probabilidad de retención de un pez de longitud $l$, a través de la grilla con separación $(d)$ se modela como:

$$
P_{l, d}=\frac{1}{N_{l}} * \sum_{i=1}^{N_{l}} \varphi_{i, l, d} \operatorname{con} \begin{array}{lll}
\varphi_{l, d}=0 & \text { si }\left(A_{l} \leq d\right) & \text { (escapado) } \\
\varphi_{l, d}=1 & \text { si }\left(A_{l}>d\right) & \text { (retenido) }
\end{array}
$$

donde $A_{l}$ es obtenido en forma aleatoria de un conjunto de valores de $A$ para una determinada $l$ y $N_{l}$ es la cantidad de peces de longitud $l$ que entran al arte.

A modo de ejemplo, se aplica el procedimiento a los datos de la pesquería industrial de merluza común (Merluccius gayi gayi) y se determina una separación inter-barra de la rejilla de $47 \mathrm{~mm}$. Con esto se lograría una longitud de captura $\left(l_{50}\right)$ de $390 \mathrm{~mm}$, una longitud de reclutamiento al arte $\left(l_{25}\right)$ de $381 \mathrm{~mm}$ con un estrecho rango de selección $(R S=18,25$ $\mathrm{mm}$ ). Finalmente, se discuten las implicancias técnicas y biológicas de la implementación del sistema en la pesquería industrial de merluza común.

Palabras claves: selectividad, redes de arrastre, sistemas de grilla selectiva (SGS), procedimiento Bootstrap, Merluccius gayi gayi.

\section{Methods to determine the inter-bar distance and the theoretical selection curve of grid sorting systems of trawlnets to fish} \begin{abstract}
through the grid with separation $(d)$ is described by:

$$
P_{l, d}=\frac{1}{N_{l}} * \sum_{i=1}^{N_{l}} \varphi_{i, l, d} \text { with } \begin{array}{lll}
\varphi_{l, d}=0 & \text { if }\left(A_{l} \leq d\right) & \text { (escaped) } \\
\varphi_{l, d}=1 & \text { if }\left(A_{l}>d\right) & \text { (retained) }
\end{array}
$$
\end{abstract}

ABSTRACT. This paper proposes a numeric procedure, based on bootstrap simulations, in order to determine the theoretical size-selection curve of grid sorting systems (GSS) in trawlnets to fish. Also, based on the size structure of the captures gotten without the device, it proposes a method in order to determine the inter-bar distance of this device. The procedure considers that the maximal width $(A)$ of the fish determine if this escapes or not. Also, any particular distribution for the maximal width of a $l$-length fish is not assumed. The probability that $l$-length fish do not pass 
where $A_{l}$ is obtained at random, of a set of values of $A$ for a particular $l$, and $N_{l}$ is the amount of fish of length $l$ that enters to the net.

To manner of example, this procedure is apply to Chilean hake (Merluccius gayi gayi) industrial fishery data and an inter-bar distance of $47 \mathrm{~mm}$ is determined. With this would be obtained a length of capture $\left(l_{50}\right)$ of $390 \mathrm{~mm}$, a length of recruitment to the net $\left(l_{25}\right)$ of $381 \mathrm{~mm}$ with a short selection range $(S R=18.25 \mathrm{~mm})$. Finally, the technical and biological implication of the implementation of this system, in the industrial fishery of Chilean hake, is discussed.

Key words: selectivity, trawlnet, grid sorting system (GSS), bootstrap procedure, Merluccius gayi gayi.

\section{INTRODUCCIÓN}

Que los sistemas de pesca sean selectivos a la talla, ya no es sólo una necesidad para la administración pesquera, sino que también representan un requerimiento para las empresas involucradas en la explotación de recursos pesqueros, particularmente para los destinados a consumo humano directo. Las crecientes exigencias de los mercados internacionales han motivado en la industria la búsqueda de sistemas de selección a la talla efectivos, con el objeto de satisfacer la demanda de ciertos calibres en los productos, y de paso aumentar la eficiencia de sus embarcaciones y reducir los costos operacionales que involucra el descarte.

La historia de las grillas (rejillas) como mecanismos selectivos comienza en Noruega, a principios de 1989, cuando la grilla Nordmore fue introducida como un sistema para reducir la captura de fauna acompañante (bycatch) en la pesca de arrastre dirigida al camarón, principalmente Gadus morhua y Melanogrammus aeglefinus. Posteriormente, las investigaciones se orientaron al diseño de un sistema que permitiera la selección a la talla de ciertas especies de peces demersales, con el objeto de evitar la captura de ejemplares juveniles. Así nace el sistema noruego Sort-X (Fig. 1a) que alcanza su desarrollo definitivo en 1991/92. Paralelamente a estas investigaciones, científicos rusos desarrollaron un sistema de grilla simple, para redes de cuatro paneles, denominado Sort-V (Isaksen, 1998b).

Con el transcurso del tiempo, los dispositivos mencionados fueron haciéndose más populares y se fueron incorporando en forma voluntaria u obligatoria en varias pesquerías de otros países. Esta transferencia de tecnología obligó a adecuar los dispositivos, en menor o mayor grado, a las realidades de cada pesquería, y en algunos casos, potenció la creación de nuevos diseños, como la grilla canadiense Nordm申re o las grillas argentinas DISELA II y DEJUPA (Fig. 1b).

Los dispositivos orientados a la selección por tallas de peces se ubican en el túnel de la red y tie- nen incorporado una sección de tela extra para permitir la fácil unión a la red. Están confeccionados con materiales rígidos, como acero o fierro y poseen un cierto ángulo de ataque que les permite mantener equilibrado el sistema y exponer el área de selección a los peces (Fig.1A y 1B).

El principio de trabajo de estos dispositivos es bastante simple y consiste en permitir que los peces pequeños pasen entre las barras de las parrillas y escapen; siendo fundamentalmente el tamaño de los peces que pasan, dependiente de la distancia (o separación) existente entre las barras.

Según Larsen e Isaksen (1993), Larsen (1996) y Ercoli et al. (1997) las ventajas de estos sistemas está en que los peces se seleccionan en una etapa muy temprana del proceso de captura, su capacidad es superior a la de los copos convencionales (malla romboidal), el proceso de selección no se ve afectado por el tamaño de las capturas o la velocidad de arrastre, no existen deformaciones debido a la tensión del arrastre y el enredo de peces es bajo, eliminando el efecto de obstrucción de la grilla. Todo esto hace que el sistema de filtración mediante grillas rígidas sea muy cercano a la situación ideal y consiga evitar un efecto similar al de distorsión de la malla y obstrucción del copo (Pikitch et al., 1995).

No obstante, cuando los sistemas se comienzan a utilizar por primera vez en una pesquería, subsisten las preguntas: ¿cuál debiera ser la separación inter-barra adecuada para permitir la retención de peces superiores a un cierto tamaño, y en qué porcentaje serán retenidos estos peces?, o más generalmente ¿cuál será el esquema selectivo para una separación inter-barra cualquiera?

Este cuestionamiento es de vital importancia cuando se pretende implementar el sistema y se requiere evitar gastos innecesarios de prueba y error. En el presente artículo, primero se desarrolla un método numérico estructural (Fryer y Sheperd, 1993) para dar respuesta a estas preguntas, luego se aplica el procedimiento a datos de merluza común (Merluccius gayi gayi) y, finalmente, se discuten las 
implicancias técnicas y biológicas de la aplicación de los sistemas a la pesquería de arrastre de merluza común.

\section{MODELO}

El planteamiento que a continuación se describe es teórico, y se fundamenta en los procesos selectivos que ocurren en los dispositivos selectivos anteriormente descritos. Si bien el modelo debe ser validado con ensayos experimentales, el procedimiento se sustenta sobre la base de grabaciones de imágenes submarinas captadas por el Instituto Nacional de Investigación y Desarrollo Pesquero (INIDEP, Argentina) sobre la merluza argentina (Merluccius hubbsi) y por el Institute of Marine Research (IMR, Noruega) sobre la merluza de Namibia (Merluccius capensis), en el sentido de que las merluzas poseen un comportamiento pasivo en el túnel de la red y no utilizan las oportunidades de escape que se le presentan (Isaksen, 1998a).

Sea $N_{l}$ el número de individuos en el intervalo de longitud cuya marca de clase es $l$ que entran al arte; $A_{l}$ los posibles valores de ancho máximo de un pez de longitud $l$ y $d$ la separación inter-barra de la rejilla del dispositivo. Finalmente, sea $\varphi_{l, d}$ una condición que evalúa el escape o retención de un pez de longitud $l$ intentando pasar a través del dispositivo con separación inter-barra, $d$.
Como lo indican Millar y Walsh (1992), $\varphi_{l, d}$ es una variable aleatoria binomial y puede tomar solamente 2 valores posibles, i.e. si el pez es retenido, $\varphi_{l, d}=1$; en tanto que si el pez escapa, $\varphi_{l, d}=0$. El escape o retención del pez depende, entre otros factores, de la longitud $(l)$ que posea, pero más realmente, depende del ancho del mismo. En este caso el ancho máximo (A). Peces de una misma longitud, $l$, pueden presentar diferentes valores de $A$, por tanto, peces de una misma longitud pueden ser retenidos o escapar del arte de pesca.

Para el caso de las grillas selectivas, la condición de escape-retención del pez puede ser modelada de múltiples formas cuando se tienen antecedentes del grado de contracción del pez, comportamiento, capacidad natatoria, flujo de agua por las grillas, luminosidad y nivel de captura en el túnel. No obstante, según lo señalado al principio de este capítulo y en la enumeración de las ventajas de estos dispositivos, muchos de estos factores no tienen incidencia en el escape-retención de las merluzas. Por tanto, en el presente trabajo, al igual que Tokai et al. (1996), se asume que la única variable que afecta el escape-retención en la rejilla es el ancho máximo del pez, aunque se reconoce que el flujo de agua por las rejillas y un mayor grado de contracción de los peces inciden en la selección. Estos últimos aspectos no han sido considerados en el modelo, porque son difíciles de medir y/o incorporar en un modelo más general.

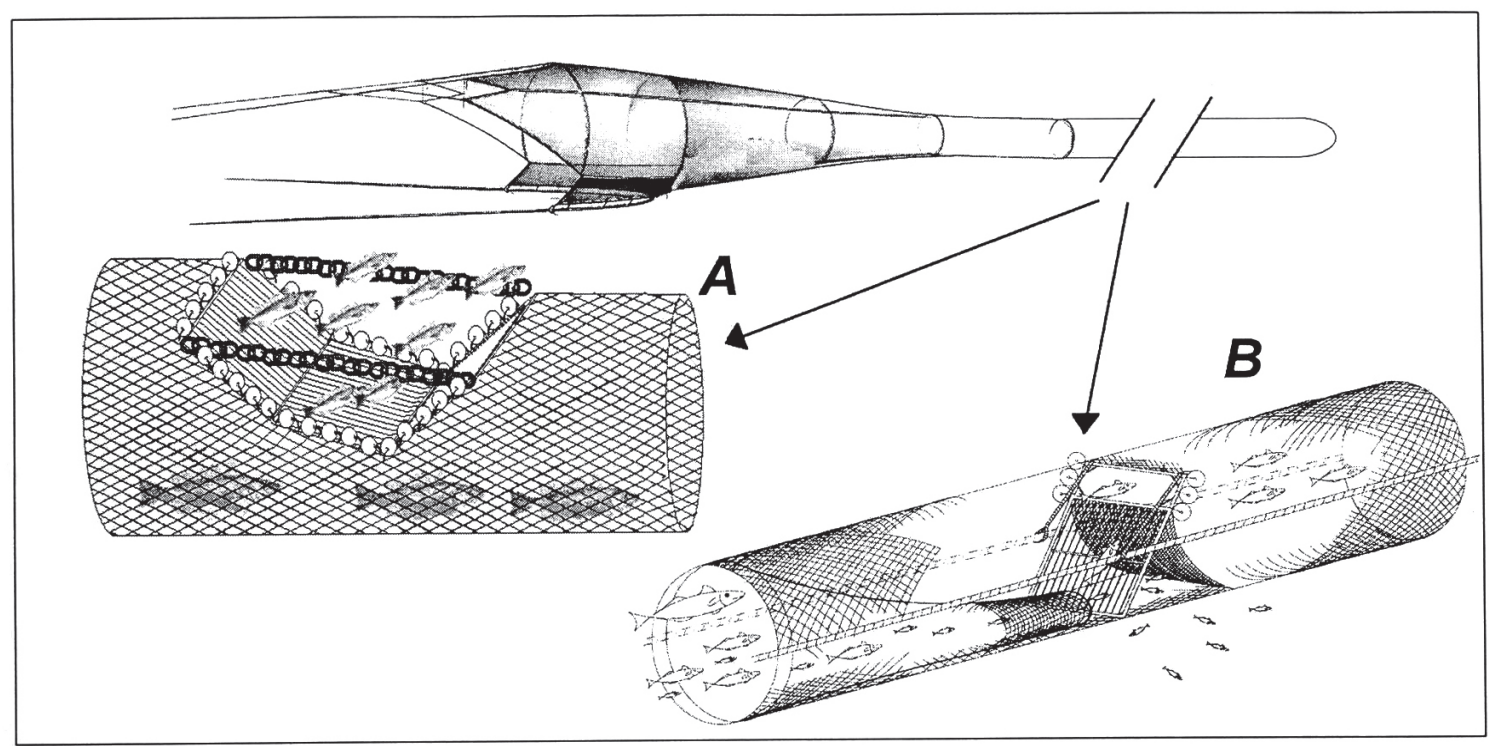

Figura 1. Dispositivos de grilla selectiva y su ubicación en la red de arrastre. (A) Sort-X y (B) DEJUPA. Figure 1. Sorting grid devise and its position in the trawlnet. Sort-X (A) and DEJUPA (B). 
Adicionalmente, un segundo supuesto que es necesario efectuar es que «la totalidad de los peces que entran al arte tienen contacto o interactúan con las grillas». Para el caso particular del DEJUPA, y en base a las observaciones submarinas disponibles, este es un supuesto que se acerca bastante a la realidad (Ercoli com. pers.). Sobre la base de estas consideraciones se evaluó la condición de escape-retención como:

$$
\varphi_{l, d}\left\{\begin{array}{lll}
0 & \text { si } A_{l} \leq d & \text { (escape) } \\
1 & \text { si } A_{l}>d & \text { (retención) }
\end{array}\right.
$$

Es decir, el pez escapa si su ancho máximo $\left(A_{l}\right)$ es inferior o igual a la separación inter-barra del dispositivo. Por otro lado, el pez es retenido si su ancho máximo es superior a dicha separación.

Como ya se mencionó, en peces de una misma longitud es posible esperar diferentes valores de ancho máximo. Luego, para evaluar la condición (1) a una talla dada, los valores de $A_{l}$ deben ser escogidos al azar y con reemplazo, desde una muestra de peces de longitud $l$.

Por otro lado, para obtener la probabilidad de retención de una talla $l$, la condición en (1) debe ser evaluada tantas veces como individuos se supone que entran de esa talla al arte, es decir, $N_{l}$. Según esto, la probabilidad de retención para una talla particular y para una separación inter-barra $(d)$, será:

$$
P_{l, d}=\frac{1}{N_{l}} * \sum_{l=1}^{N_{l}} \varphi_{i, l, d}
$$

donde $\varphi_{i, l, d}$ debe ser evaluado $i$-veces como en (1).

Luego, para determinar las proporciones de retención a la talla, es necesario solamente medir el ancho máximo para varios peces de longitud $l, l+l$, $l+2, \ldots, l+n$, siendo $n$ el número de clases de tamaño que se pretende evaluar. Por otro lado, los valores de $N_{l}$ pueden ser simulados u obtenidos de las distribuciones de frecuencias de tallas de las capturas.

Una vez determinados los porcentajes de retención a la talla para una distancia inter-barra particular $\left(P_{l, d}\right)$, es posible ajustar un modelo a dichos valores con el fin de determinar el esquema selectivo. En este punto es posible considerar una gran variedad de modelos, como la típicas funciones logita y probita (Pope et al., 1975), sus funciones inversas o una curva de selección asimétrica, como la de Richard (Millar, 1993), es decir, cualquier fun- ción no-decreciente que tome valores entre 0 y 1 .

En este trabajo se eligió la curva de selección de forma logística (Millar y Walsh, 1992),

$$
r(l)=\frac{e^{(a+b l)}}{1+e^{(a+b l)}}
$$

la cual es la inversa de la función logita con parámetros $a$ y $b$.

Luego de parametrizar y ajustar la ecuación a los datos de proporciones de retención, es posible derivar las longitudes de retención $\left(l_{25}, l_{50}\right.$ y $\left.l_{75}\right)$, el rango de selección $(R S)$ y el factor de selección $(F S)$.

$$
\begin{aligned}
& l_{25}=\frac{\ln (1 / 3)-a}{b} \\
& l_{50}=\frac{-a}{b} \\
& l_{75}=\frac{\ln (3)-a}{b} \\
& R S=l_{75}-l_{25} \\
& F S=l_{50} / d
\end{aligned}
$$

Determinadas las proporciones de retención a la talla y el esquema selectivo, para diferentes separaciones entre barras, se hace necesario discriminar cuál es la distancia inter-barra adecuada para los objetivos que se persigan. El procedimiento más adelante presentado es útil tanto para la administración de pesquerías como para una empresa pesquera en particular.

Sin embargo, es necesario destacar que los objetivos que persigue la administración pesquera y los que persigue la industria, no son necesariamente los mismos. Por lo tanto, la idea que subyace a la determinación de la separación inter-barra adecuada, es proporcionar una tabla de decisión de doble entrada, en la que el administrador seleccione la talla mínima de captura del pez y el porcentaje (en número) bajo esta talla que desea que se capture. Dados estos datos, se obtiene de la tabla la distancia inter-barra que satisface estas condiciones.

Para graficar la construcción de la tabla de decisión, y a modo de ejemplo, considere la evaluación de 3 valores de separación inter-barra, es decir, $d l$, $d 2$ y $d 3$. Además, considere que se requiere evaluar la retención en individuos de longitudes $l 1, l 2, l 3$ y 14. 
Ahora, sea $f_{l}$ la frecuencia observada a la talla $l$ (o la marca de clase $l$ ), obtenida a partir de las distribuciones de frecuencias de tallas de las capturas y $P_{l, d 1}, P_{l, d 2}$ y $P_{l, d 3}$ los vectores de probabilidad de retención a la talla al considerar separaciones interbarra $d 1, d 2$ y $d 3$, respectivamente.

El porcentaje de individuos bajo la talla $l 1$, al considerar una separación inter-barra $d l$ es:

$$
\% B T_{l l, d l}=\frac{\sum_{l \min }^{l 1} f_{l} * P_{l, d l}}{\sum_{l \min }^{l \operatorname{má} x} f_{l} * P_{l, d l}}
$$

Luego, el porcentaje de individuos bajo la talla $l 2$, al considerar una separación inter-barra $d 3$, es

$$
\% B T_{l 2, d 3}=\frac{\sum_{l \min }^{l 2} f_{l} * P_{l, d 3}}{\sum_{l \min }^{l \operatorname{má} x} f_{l} * P_{l, d 3}}
$$

más generalmente, el porcentaje de retención de individuos bajo la talla $l x$, al considerar una separación inter-barra $d y$, es

$$
\% B T_{l x, d y}=\frac{\sum_{l \min }^{l x} f_{l} * P_{l, d y}}{\sum_{l \min }^{l \operatorname{máx}} f_{l} * P_{l, d y}}
$$

donde lmín es la marca de clase del intervalo de longitud mínimo observada en la distribución de fre- cuencias de tallas de las capturas y lmáx es la marca de clase del intervalo de longitud máximo observado.

Dado que los valores que se obtienen en la ecuación (2) están condicionados a las elecciones azarosas y con reemplazo efectuadas según el criterio en (1), es posible obtener múltiples valores de $P_{l, d}$. A partir de estos registros de $P_{l, d}$ es posible calcular el promedio y la medida de dispersión que se desee, así como los respectivos intervalos de confianza, siendo este procedimiento un enfoque paramétrico del método bootstrap (Efron, 1981, 1985). Lo mismo se aplica al \%BT.

\section{APLICACIÓN A MERLUZA COMÚN}

\section{Antecedentes}

En Chile, el mayor número de estudios directos de selectividad se han efectuado precisamente en merluza común (Saetersdal y Villegas, 1968; Arana, 1970; Klenner, 1978; Pavez, 1981; Gálvez et al., 1999). Todos estos estudios se han efectuado para evaluar el esquema selectivo de las redes utilizadas por la flota, considerando diferentes tamaños de malla en el copo, por lo que no se dispone de antecedentes de estudios directos relativos al esquema de selección de dispositivos de rejilla en la pesquería de merluza común.

No obstante lo anterior, algunos dispositivos de grilla se han aplicado en pesquerías de especies similares tales como Merluccius hubbsi (Ercoli et al., 1997, 1998; Anónimo, 1998), M. capensis (Ercoli, com. pers.), Gadus morhua y Melanogrammus aeglefinus (Isaksen, 1996, 1998b; Larsen e Isaksen, 1993), entre otros, pero a pesar de esto siguen siendo escasos los trabajos publicados a la fecha. Debido a la similitud de M. gayi gayi con $M$. hubbsi, se entrega en la Tabla 1 un resumen de los principales

Tabla 1. Principales resultados obtenidos en experimentos con sistemas de grilla selectiva en Merluccius hubbsi. Table 1. Principal results obtained by experiments with grid sorting systems in Merluccius hubbsi.

\begin{tabular}{|lccccc|}
\hline \multicolumn{1}{|c}{ Autor } & \multicolumn{3}{c}{ Ercoli et al. (1998) } & \multicolumn{2}{c|}{ Hafi (1998) } \\
\hline Separación inter-barra $(\mathbf{m m})$ & 30 & 33 & 40 & 34 & 40 \\
\hline$L_{50}(\mathrm{~mm})$ & 287 & 320 & 374 & 333 & 396 \\
$F S$ & 9,56 & 9,70 & 9,35 & 9,79 & 9,90 \\
$R S(\mathrm{~mm})$ & 60 & 67 & 100 & 54 & 56 \\
Captura por lance $(\mathrm{kg})$ & $115-929$ & $511-1.067$ & $427-641$ & $1.750-2.706$ & $1.363-5.255$ \\
Sistema & DEJUPA & DEJUPA & DEJUPA & SORT-X & SORT-X \\
\hline
\end{tabular}


resultados de estudios efectuados con sistemas de grilla en esta última especie.

\section{Fuente de información}

Para aplicar el procedimiento descrito a merluza común, durante enero de 1998 se recolectaron a bordo de embarcaciones industriales 160 ejemplares, cuyas longitudes totales estuvieron comprendidas entre $300 \mathrm{~mm}$ y $450 \mathrm{~mm}$ (10 individuos por cada talla). A estos ejemplares se les midió la longitud total ( $L T$ ) y ancho máximo (a la altura de la primera aleta dorsal), considerando que esta medida morfométrica del recurso es la que incide directamente en el escape de los peces por las rejillas del sistema. Las capturas, de donde se obtuvieron los ejemplares muestreados, se efectuaron con redes de arrastre de fondo de dos paneles tipo «Engel» con luz de malla estirada en el copo de 100 $\mathrm{mm}$.

Con el objeto de disponer de datos de frecuencias a la talla, $f_{l}$, se utilizaron los registros consignados por Gálvez et al. (1998) y que fueron obtenidos a partir de datos colectados a bordo de embarcaciones industriales (Fig. 2). Este histograma representa la distribución de frecuencias de tallas del stock explotable de merluza común en la zona centro-sur de Chile, durante 1997.

\section{RESULTADOS}

A pesar que el procedimiento desarrollado no requiere de la modelación de la relación entre longitud total y ancho máximo, se ajustaron ecuaciones lineales y no-lineales simples a estos datos, con el objeto de caracterizar dicha relación. Se observó una correlación positiva entre $L T$ y $A$, re-

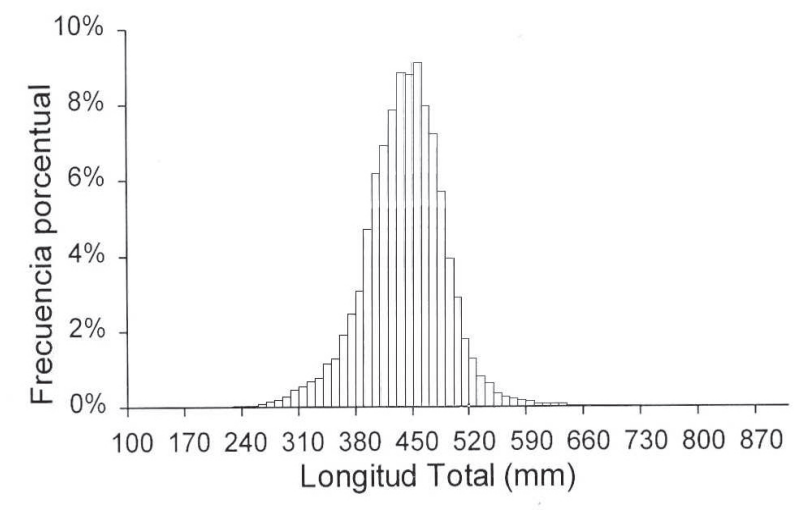

Figura 2. Distribución de frecuencias de tallas del stock de Merluccius gayi gayi en 1997.

Figure 2. Size-frequency distribution of Merluccius gayi gayi stock, in 1997. sultando $A$ estadísticamente mejor explicado a través de un modelo potencial con un $r^{2}$ de $88 \%$ (Fig. 3).

Como se mencionó anteriormente, se evaluó en forma teórica el efecto selectivo que podrían tener los dispositivos de grillas metálicas rígidas en merluzas comprendidas entre 300 y $450 \mathrm{~mm}$ de longitud total, ya que se consideró este rango de interés para el estudio, desde el punto de vista de la empresa y de la administración pesquera. Adicionalmente, se consideraron 8 separaciones inter-barra, las que estuvieron comprendidas entre 41 y $55 \mathrm{~mm}$ (cada $2 \mathrm{~mm}$ ).

Para cada longitud total y separación inter-barra se evaluó la ecuación (2) con un $N$, que dependió de la frecuencia porcentual de individuos a esa talla $\left(f_{l}\right)$, según lo establecido por Gálvez et al. (1998) y un valor arbitrario en este caso de $1.000\left(N_{l}=f_{l}^{*} 1.000\right)$. Los resultados de probabilidad o porcentaje de retención a la talla para cada separación inter-barra se muestran en la Fig. 4.

Se observó que, para todas las $d$ consideradas, las probabilidades de retención alcanzaron el valor 1, con excepción de la separación inter-barra de $55 \mathrm{~mm}$, en que se alcanzó una probabilidad de retención máxima de 0,90 a una longitud total de $450 \mathrm{~mm}$. No obstante, es razonable pensar que para $L T$ superiores a $450 \mathrm{~mm}$ las proporciones de retención sean cercanas a 1 , para una separación $d=55 \mathrm{~mm}$.

Para modelar el esquema selectivo, a las proporciones de retención se le ajustó la ecuación (3) mediante el programa computacional Jandel Scientific, utilizando la opción de mínimos cuadrados no lineales a través del algoritmo de LevenburgMarquardt. Los resultados de este ajuste se muestran en la Figura 4 y Tabla 2. Se puede apreciar a través del coeficiente de determinación que los ajustes, en el peor de los casos, explicaron más del $96 \%$ de la variabilidad. Los valores del parámetro adimensional $a$ estuvieron comprendidos entre $-68,46$ y 38,26 , en tanto que los valores de $b$ variaron entre $0,0936 \mathrm{~mm}^{-1}$ y $0,1967 \mathrm{~mm}^{-1}$. Adicionalmente, se observó que los errores estándar de los parámetros tendieron a disminuir con el aumento de la separación interbarra. 
Se observó que las longitudes al 25\%, 50\% y $75 \%$ de retención aumentaron en forma proporcional a la separación inter-barra (Tabla 2 y Fig. 5). En forma similar, el factor de selección $(F S)$ disminuyó linealmente con el aumento de la separación inter-barra. Por otro lado, el rango de selección $(R S)$ estuvo comprendido entre 17,63 mm y $21,90 \mathrm{~mm}$, con excepción del valor calculado con $d=41$ mm (Fig. 5).

Una vez establecidas las proporciones de retención a la talla y considerando las frecuencias porcentuales consignadas en la Fig. 2, se procedió a calcular el porcentaje de merluzas bajo talla (\%BT), mediante aplicar la ecuación (5). Para tal efecto, se consideraron en los análisis $l_{x}$ entre 360 y $400 \mathrm{~mm}$ de longitud total.

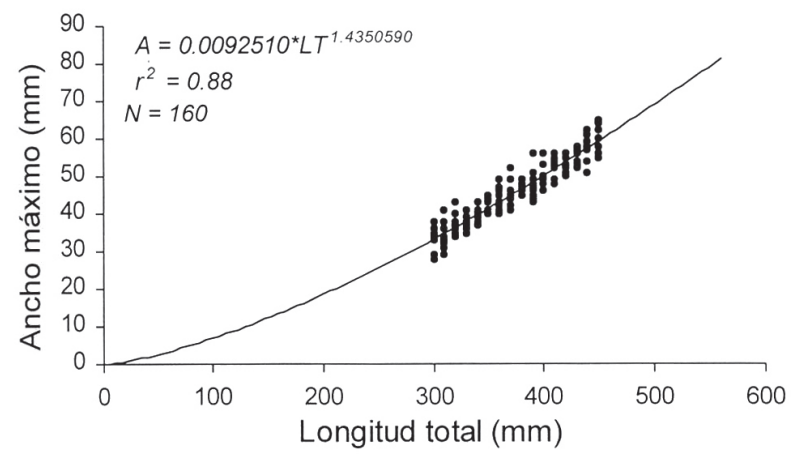

Figura 3. Relación entre longitud total y ancho máximo para Merluccius gayi gayi.

Figure 3. Relation between total length and maximum width in Merluccius gayi gayi.
Una vez hecho esto, se volvió a repetir el proceso completo un total de 100 veces, de modo de poder estimar las proporciones de retención bajo talla promedio y las respectivas desviaciones estándar de estos estimados. Con esto fue posible conformar la tabla de decisión mencionada anteriormente (Tabla 3 y Fig. 6).

Como era de esperar, se observó que los porcentajes de retención bajo talla, en cualquier talla considerada, disminuyen a medida que se incrementa la separación inter-barra, hasta ser cero. No obstante, los porcentajes bajo las tallas de $390 \mathrm{~mm}$ y $400 \mathrm{~mm}$ son asintóticos para separaciones, $d$, igual y superiores a $49 \mathrm{~mm}$ (Fig. 6). Al analizar la desviación estándar de los porcentajes promedio de retención bajo talla para diferentes separaciones entre barras (Tabla 3), es posible apreciar que para las longitudes de pez inferiores a $360 \mathrm{~mm}$ y $370 \mathrm{~mm}$ los registros disminuyen a medida que se incrementa la separación inter-barra. Esto indicaría que la estimación de \%BT promedio para estas tallas se hace más exacta a medida que se aumenta la separación interbarra. Una situación inversa ocurre al considerar las longitudes totales de 380 mm, 390 mm y 400 mm. Aquí, la desviación estándar del porcentaje de retención medio aumenta a medida se que incrementa la separación inter-barra hasta $d=47 \mathrm{~mm}$, luego las des-

Tabla 2. Resultados del ajuste de las curvas de selección para separación inter-barra entre 41 y 55 mm. Los valores en paréntesis indican errores estándar

Table 2. Results of the selection curves fits for inter-bar distance between 41 and $55 \mathrm{~mm}$. Values in parentheses are standard errors

\begin{tabular}{|lrrrrrrrr|}
\hline & \multicolumn{7}{c|}{ Separación inter-barra (mm) } \\
\cline { 2 - 10 } Parámetro & $\mathbf{4 1}$ & $\mathbf{4 3}$ & $\mathbf{4 5}$ & $\mathbf{4 7}$ & $\mathbf{4 9}$ & $\mathbf{5 1}$ & $\mathbf{5 3}$ & $\mathbf{5 5}$ \\
\hline $\boldsymbol{a}$ & $-68,46$ & $-35,95$ & $-39,84$ & $-47,00$ & $-50,04$ & $-38,26$ & $-42,75$ & $-51,50$ \\
& $(12,73)$ & $(6,41)$ & $(8,45)$ & $(7,95)$ & $(5,90)$ & $(5,66)$ & $(5,57)$ & $(4,72)$ \\
$\boldsymbol{b}\left(\mathbf{m m}^{-1}\right)$ & 0,1967 & 0,1003 & 0,1071 & 0,1204 & 0,1246 & 0,0936 & 0,1010 & 0,1198 \\
& $(0,04)$ & $(0,02)$ & $(0,02)$ & $(0,02)$ & $(0,01)$ & $(0,01)$ & $(0,01)$ & $(0,01)$ \\
$\boldsymbol{r}^{\mathbf{2}}(\boldsymbol{\%})$ & 98,28 & 96,88 & 96,10 & 97,69 & 98,83 & 97,01 & 97,25 & 98,51 \\
\hline $\boldsymbol{l}_{\mathbf{2 5}}(\mathbf{m m})$ & 342 & 347 & 362 & 381 & 393 & 397 & 412 & 421 \\
$\boldsymbol{l}_{\mathbf{5 0}}(\mathbf{m m})$ & 348 & 358 & 372 & 390 & 401 & 409 & 423 & 430 \\
$\boldsymbol{l}_{\mathbf{7 5}}(\mathbf{m m})$ & 354 & 369 & 382 & 400 & 410 & 420 & 434 & 439 \\
$\boldsymbol{F S}$ & 8,49 & 8,33 & 8,27 & 8,31 & 8,19 & 8,01 & 7,98 & 7,82 \\
$\boldsymbol{R S}(\mathbf{m m})$ & 11,17 & 21,90 & 20,52 & 18,25 & 17,63 & 23,47 & 21,75 & 18,34 \\
\hline
\end{tabular}


viaciones presentan una tendencia a la baja. Para estas longitudes totales, esto sugiere que los porcentajes bajo talla presentan mayor variabilidad cuando se considera una separación inter-barra de $47 \mathrm{~mm}$ (Tabla 3).

$\mathrm{Al}$ analizar la desviación estándar de los porcenta-

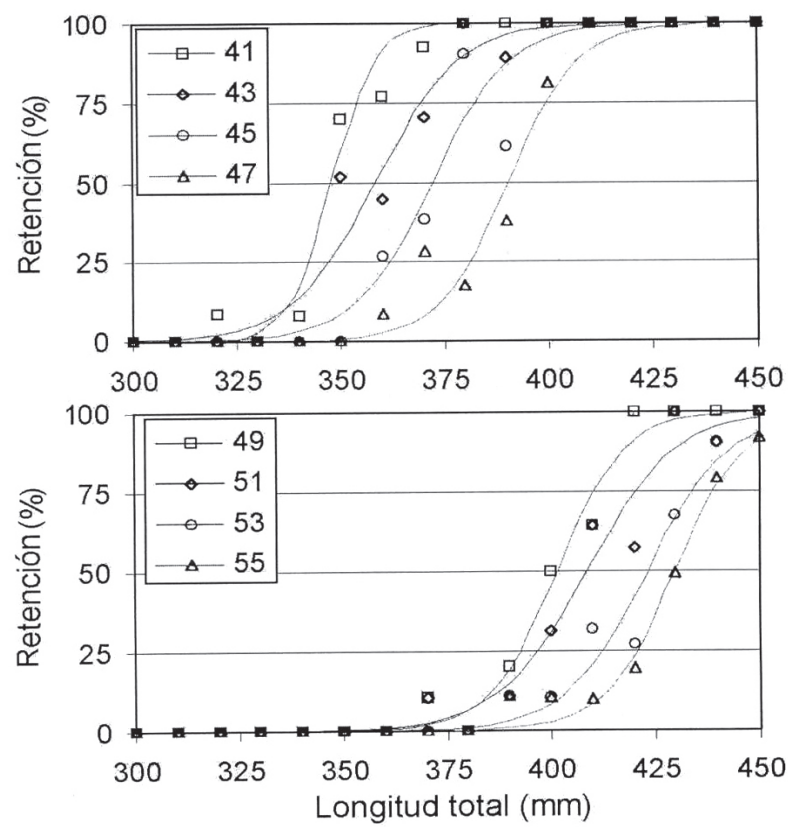

Figura 4. Porcentajes de retención a la talla y curvas de selección para separación inter-barra entre 41 y $55 \mathrm{~mm}$.

Figure 4. Size-retention ratio and selection curves to interbar distance between 41 and $55 \mathrm{~mm}$. jes de retención promedio, a una misma separación inter-barra y para las diferentes longitudes de retención (es decir, registros en las columnas de la Tabla 3), es posible observar que la desviación estándar del porcentaje de retención aumenta a medida que se aumenta la talla considerada. Esto se ve corroborado al graficar las distribuciones de probabilidad de los porcentajes de retención para diferentes tallas y para una separación inter-barra particular, en este caso $d=47 \mathrm{~mm}$ (Fig. 7). En esta figura se aprecia que el rango de porcentajes bajo talla es más amplio a medida que se aumenta la longitud de retención. Esto se debe a que los peces de mayor longitud total registran una mayor variación en el ancho máximo que los peces pequeños, lo cual se debe probablemente a las diferencias en su condición reproductiva y/o a aspectos relacionados con la alimentación de los individuos.

Para seleccionar la separación inter-barra adecuada a la pesquería, se adoptó la posición y objetivos de la administración pesquera. Según esto y considerando que la talla de primera madurez sexual de merluza común es de $370 \mathrm{~mm}$ (Alarcón y Arancibia, 1993), la separación interbarra adecuada se determinó en $47 \mathrm{~mm}$. Con esta separación el dispositivo retendría solamente el $0,23 \%$ (desv.est. $=0,037 \%$ ), en número, de los ejemplares inferiores a $370 \mathrm{~mm}$ de longitud total, en contraste con el porcentaje de retención

Tabla 3. Porcentaje actual de la captura bajo talla de Merluccius gayi gayi y estimación del mismo según separación inter-barra. Los valores en paréntesis indican la desviación estándar.

Table 3. Current percentage of the catch under size of Merluccius gayi gayi and this estimation for different inter-bar distances. Values in parentheses are standard deviance

\begin{tabular}{|c|c|c|c|c|c|c|c|c|c|}
\hline \multirow{2}{*}{$\begin{array}{l}\text { Longitud } \\
\text { de } \\
\text { retención }\end{array}$} & \multicolumn{9}{|c|}{ Separación inter-barra (mm) } \\
\hline & Sin rejilla & 41 & 43 & 45 & 47 & 49 & 51 & 53 & 55 \\
\hline$<360 \mathrm{~mm}$ & $5,69 \%$ & $\begin{array}{r}1,14 \% \\
(0,039 \%)\end{array}$ & $\begin{array}{r}0,69 \% \\
(0,032 \%)\end{array}$ & $\begin{array}{r}0,00 \% \\
(0,000 \%)\end{array}$ & $\begin{array}{r}0,00 \% \\
(0,000 \%)\end{array}$ & $\begin{array}{r}0,00 \% \\
(0,000 \%)\end{array}$ & $\begin{array}{r}0,00 \% \\
(0,000 \%)\end{array}$ & $\begin{array}{r}0,00 \% \\
(0,000 \%)\end{array}$ & $\begin{array}{r}0,00 \% \\
(0,000 \%)\end{array}$ \\
\hline$<370 \mathrm{~mm}$ & $7,58 \%$ & $\begin{array}{r}2,74 \% \\
(0,052 \%)\end{array}$ & $\begin{array}{r}1,72 \% \\
(0,056 \%)\end{array}$ & $\begin{array}{r}0,64 \% \\
(0,044 \%)\end{array}$ & $\begin{array}{r}0,23 \% \\
(0,037 \%)\end{array}$ & $\begin{array}{r}0,00 \% \\
(0,000 \%)\end{array}$ & $\begin{array}{r}0,00 \% \\
(0,000 \%)\end{array}$ & $\begin{array}{r}0,00 \% \\
(0,000 \%)\end{array}$ & $\begin{array}{r}0,00 \% \\
(0,000 \%)\end{array}$ \\
\hline$<380 \mathrm{~mm}$ & $10,02 \%$ & $\begin{array}{r}5,05 \% \\
(0,067 \%)\end{array}$ & $\begin{array}{r}3,56 \% \\
(0,081 \%)\end{array}$ & $\begin{array}{r}1,74 \% \\
(0,080 \%)\end{array}$ & $\begin{array}{r}1,10 \% \\
(0,083 \%)\end{array}$ & $\begin{array}{r}0,31 \% \\
(0,044 \%)\end{array}$ & $\begin{array}{r}0,34 \% \\
(0,048 \%)\end{array}$ & $\begin{array}{r}0,00 \% \\
(0,000 \%)\end{array}$ & $\begin{array}{r}0,00 \% \\
(0,000 \%)\end{array}$ \\
\hline$<390 \mathrm{~mm}$ & $13,08 \%$ & $\begin{array}{r}8,28 \% \\
(0,064 \%)\end{array}$ & $\begin{array}{r}6,86 \% \\
(0,078 \%)\end{array}$ & $\begin{array}{r}4,82 \% \\
(0,089 \%)\end{array}$ & $\begin{array}{r}1,83 \% \\
(0,113 \%)\end{array}$ & $\begin{array}{r}0,31 \% \\
(0,044 \%)\end{array}$ & $\begin{array}{r}0,34 \% \\
(0,048 \%)\end{array}$ & $\begin{array}{r}0,00 \% \\
(0,000 \%)\end{array}$ & $\begin{array}{r}0,00 \% \\
(0,000 \%)\end{array}$ \\
\hline$<400 \mathrm{~mm}$ & $17,80 \%$ & $\begin{array}{r}13,26 \% \\
(0,061 \%)\end{array}$ & $\begin{array}{r}11,42 \% \\
(0,105 \%)\end{array}$ & $\begin{array}{r}7,97 \% \\
(0,152 \%)\end{array}$ & $\begin{array}{r}4,05 \% \\
(0,168 \%)\end{array}$ & $\begin{array}{r}1,53 \% \\
(0,125 \%)\end{array}$ & $\begin{array}{r}0,99 \% \\
(0,105 \%)\end{array}$ & $\begin{array}{r}0,74 \% \\
(0,105 \%)\end{array}$ & $\begin{array}{r}0,81 \% \\
(0,116 \%)\end{array}$ \\
\hline
\end{tabular}


actual ( $\sin$ rejilla) de 7,58\%. Para esta separación entre barras la longitud de captura al $50 \%$ es de 390 mm de $L T$, en tanto que la talla de reclutamiento a la pesquería es de $381 \mathrm{~mm}$ de $L T$.

\section{DISCUSIÓN}

\section{Sobre las implicancias técnicas}

Según Larsen (1996) la construcción del sistema SORT-X está basada en experimentos prácticos y en observaciones submarinas, a la vez que se han efectuado experiencias en Gadus morhua, Melanogrammus aeglefinus, Pollachus virens, Sebastes spp y Reinhardtius hippoglossoides, y se

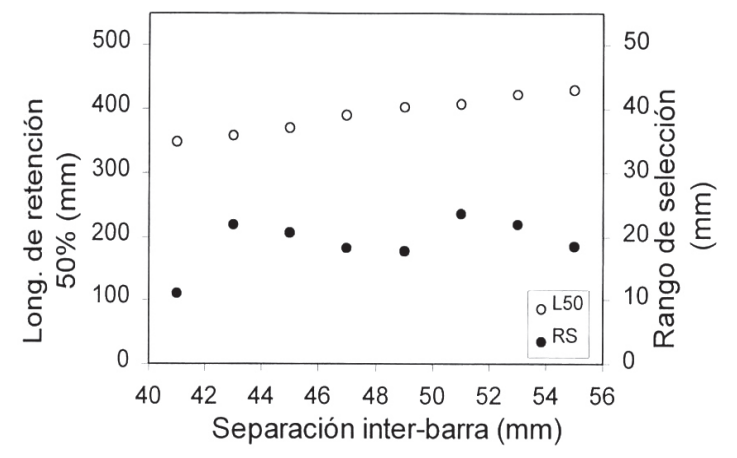

Figura 5. Relación entre distancia inter-barra, longitud de primera captura y rango de selección.

Figure 5. Relationship between inter-bar distance, length of first catch and selection range.

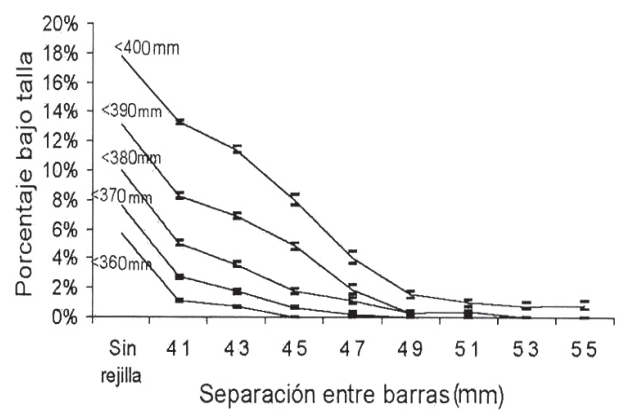

Figura 6. Porcentaje (en número) de las capturas bajo talla, para el arte de pesca operando sin rejilla y con rejilla de separación inter-barra entre 41 y $55 \mathrm{~mm}$. Las líneas cortas indican el valor máximo y mínimo.

Figure 6. Percentage (in number) of catch under size for the trawlnet without grid and with grid of 41 to 55 mm inter-bar distance. The short lines are de minimum and maximum values. ha alcanzado en forma "filo de cuchillo" la selección de la talla deseada, para todas las especies examinadas. Por otro lado, según Ercoli et al. (1997, 1998) y Anónimo (1998) resultados similares se han encontrado en experiencias con DEJUPA y SORT$\mathrm{X}$ en la pesquería de Merluccius hubbsi. Estos son argumentos válidos para suponer, al menos, que este tipo de dispositivos podría ser implementado con éxito en la pesquería industrial de arrastre de merluza común, dada la similitud de los artes de pesca empleados y del comportamiento de la especie.

Según los antecedentes disponibles de los sistemas, el proceso de selectividad se ve poco afectado por el tamaño de las capturas y por las velocidades de arrastre. A este respecto, el proveedor del dispositivo SORT-X indica que en las experiencias realizadas durante septiembre de 1991, no se observaron cambios drásticos en las propiedades selectivas, solamente se observaron leves disminuciones de la talla de captura $\left(l_{50}\right)$ cuando se obtuvieron capturas superiores a 10 ton/hora de arrastre. Estos hechos avalan, aún más, la factibilidad de implementar el sistema en la pesquería industrial de merluza, ya que según Gálvez (1998), solamente el 34\% de los lances de pesca superan las 10 ton/hora de arrastre.

Por otro lado, se ha demostrado que en una red de arrastre de fondo, parte del escape de los peces se realiza en el sector del túnel y copo, a través de las mallas en la parte superior e inferior de éste, debido principalmente al flujo de agua filtrado a través de las mismas (Arana, 1970; Suuronen, 1995). Este flujo se vería potenciado por la inclusión del sistema de rejilla, lo cual aumentaría la frecuencia de selección, es decir, más peces serían forzados a interactuar con la rejilla.

Al realizar experiencias de arrastre de pantalón con mallas cuadradas, Isaksen (1996) observó que los rangos de selección para el Gadus morhua y Melanogrammus aeglefinus eran los mismos que los obtenidos con las rejillas del sistema SORT-X. No obstante, Omoto et al. (1998) no encontraron diferencias significativas al comparar curvas de selección con mallas cuadradas y romboidales, para varias especies. Lo anterior indica que no existe una opinión única y contundente en relación al uso de mallas cuadradas; más aún cuando no se ha evaluado el grado de saturación del copo con altas densidades de captura para este tipo malla, por lo que el uso de la rejilla tendría una ventaja comparativa.

En relación con la maniobrabilidad del sistema a bordo, la mayoría de los investigadores (Larsen, 
1996, Isaksen, 1996, Anónimo, 1996, Ercoli, com. pers.) concluyen que no se presentan problemas significativos, en barcos industriales con rampa a popa, como los que actualmente operan en la pesquería industrial de merluza común. También se menciona que la operación no es entorpecida ni obstaculizada por algún tipo de basura submarina (Ercoli, com. pers.).

Finalmente, teniendo en consideración los fundamentos básicos de la dinámica de fluidos, es posible afirmar que más agua circulará a través del arrastre con un sistema de rejillas que sin el sistema. Incrementos en el flujo de agua saliente del arte de pesca implica que este ejerce una menor resistencia al avance, lo que incrementa la eficiencia y reduce el consumo de combustible. El tamaño de las rejillas y la distancia entre barras son factores vitales que inciden en el flujo de agua que pasa a través de las rejillas. Grandes flujos de agua por las rejillas son deseables cuando se requiere incrementar las velocidades de arrastre y no aumentar la resistencia al avance.

No obstante todos los antecedentes expuestos y que guardarían algún grado de relación con la pesquería de $M$. gayi gayi, aún quedan algunas cuestiones sin resolver, y que resulta interesante considerar, si se piensa aplicar el sistema a la pesquería chilena de merluza común. Estas son:

- ¿Se produce alguna distorsión de la red delante de la rejilla, al obtener grandes capturas?

- ¿Qué se podría esperar acerca de la sobrevivencia de los peces escapados a través de la rejilla? ¿Podría ser la mortalidad de las merluzas escapadas similar a las de Gadus morhua y Melanogrammus aeglefinus, las que son nulas y muy bajas, respectivamente?

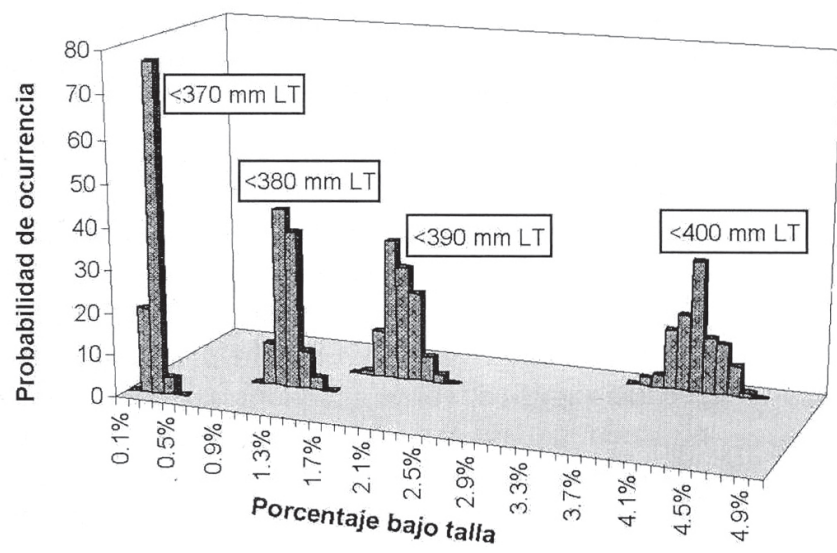

Figura 7. Distribución de probabilidad de los porcentajes de retención para diferentes tallas y para una separación inter-barra (d) de $47 \mathrm{~mm}$.

Figure 7. Probability distribution of the retention percentage for different sizes and a inter-bar distance $(d)$ of $47 \mathrm{~mm}$.
- ¿El proceso selectivo que se verifica en las mallas del copo es tan poco eficiente, como para justificar la incorporación de un sistema seleccionador de rejilla?

\section{Validación del método e implicancias biológicas}

Teniendo en consideración la declaración de Cancún (México, 1992), la cual indica que "Los Estados deben promover el desarrollo y uso de artes de pesca selectivos, promover la minimización del descarte de la captura de especies objetivo y minimizar el by-catch de las especies incidentales" y en base a los antecedentes expuestos es posible afirmar que los sistemas de grillas selectivas satisfacen plenamente los requerimientos de dicha recomendación. Por tanto, este tipo de dispositivos podría ser catalogado como una Tecnología de Pesca Responsable (García, 1996). Precisamente, según los resultados encontrados en experiencias de terreno y debido a la concepción de los sistemas, es posible afirmar que este tipo de dispositivos haría más selectivas las tradicionales redes de pesca (copo con malla romboidal) que se han implementado en la historia de la pesquería de merluza común.

Al comparar los resultados teóricos obtenidos en este estudio (Tabla 2), con los resultados empíricos obtenidos por otros autores en $M$. hubbsi (Tabla 1), es posible observar una similitud en el comportamiento de los registros. Más explícitamente, el comportamiento o tendencia de los parámetros al ser analizado versus otro parámetro es similar (Fig. 8); en efecto, las relaciones entre $l_{50}, F S, R S$ y la separación inter-barra $(d)$ presentan similares tendencias lineales para los resultados teóricos obtenidos en este estudio y para los resultados empíricos obtenidos por otros autores. La única excepción se verifica al graficar $l_{50}$ contra $F S$, en que los resultados de este estudio indican que el $F S$ disminuye a medida que aumenta la longitud de captura al 50\%, 
en tanto que los resultados de otros autores indican que el $F S$ se mantiene casi constante para las $l_{50}$ determinadas.

Si bien la validación de los resultados de este estudio debe realizarse mediante la prueba de un sistema de rejilla -con las separaciones entre barras aquí analizadas- en merluza común, lo expuesto en el párrafo anterior hace ver por analogía que el método aquí planteado es sustentable, y podría ser correctamente aplicado. Teniendo esto en consideración, es posible afirmar que la aplicación de los sistemas de rejilla en la pesquería de $M$. gayi gayi produciría efectivamente una selección cercana a la forma "filo de cuchillo", o al menos, la selección sería más pronunciada que con las mallas convencionales usadas en la pesquería.

Dado que según INPESCA (1996) los reclutamientos de merluza común no son estables en el tiempo, y pueden presentar incrementos sustancia- les de un año al otro, es posible registrar altas incidencias de ejemplares pequeños en las capturas industriales. Actualmente, la captura de estos ejemplares no puede ser evitada, debido particularmente a que las zonas y períodos de reclutamiento (Payá et al., 1995) no coinciden con los períodos de veda actuales. El problema sería subsanado mediante la incorporación de algún sistema de rejilla, asegurando evitar la consabida sobrepesca por reclutamiento. Al mismo tiempo, se permitiría el incremento en peso de estos individuos con lo cual se lograría un aumento volumétrico mayor en las capturas para igual cantidad de ejemplares capturados y se lograría, en el mediano-largo plazo, estabilizar la biomasa en niveles superiores a los actuales.

Considerando que, según Alarcón y Arancibia (1993), la merluza común posee una talla de primera madurez sexual de $370 \mathrm{~mm}$ de $L T$ y que de implementar el dispositivo con separación inter-ba-
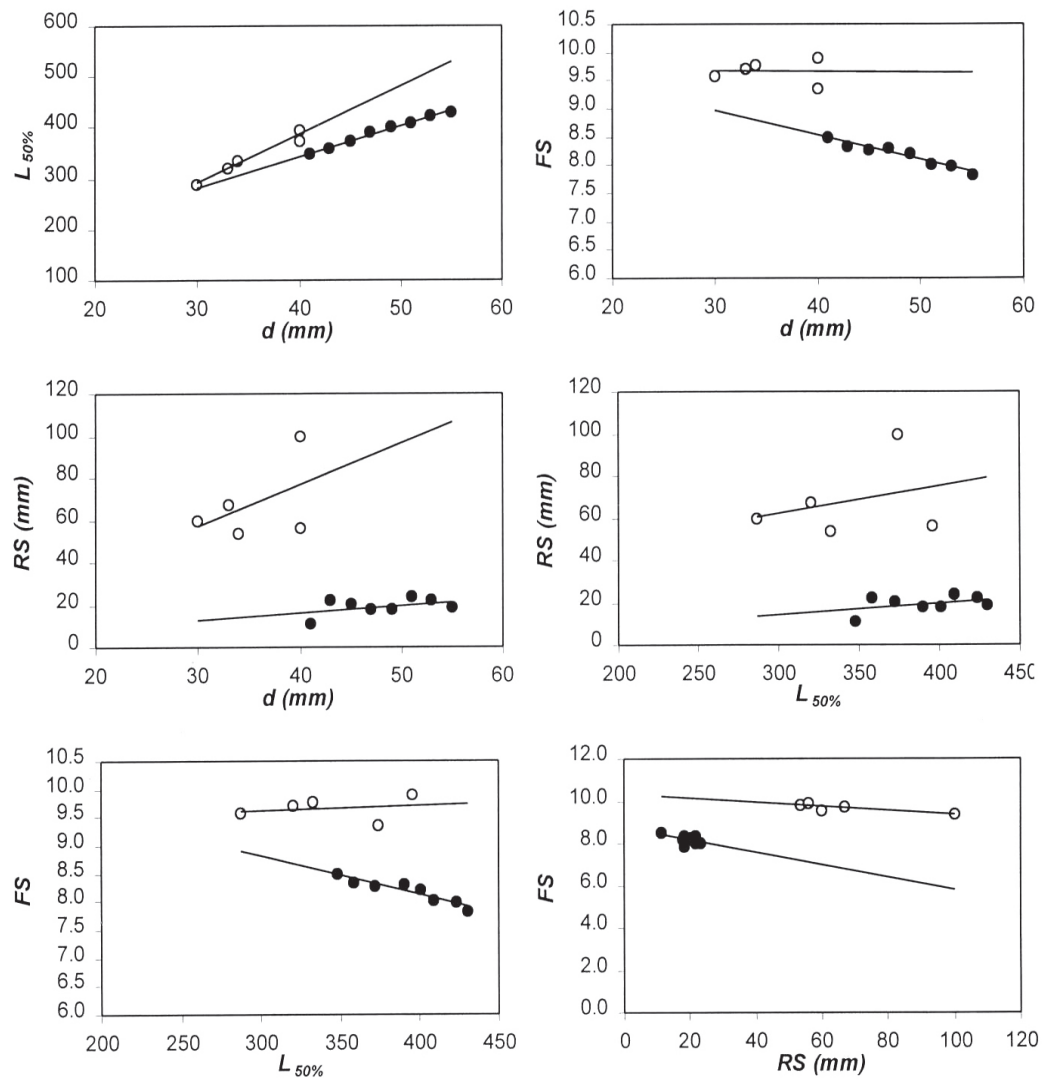

Figura 8. Comparación de las tendencias de los resultados teóricos obtenidos en Merluccius gayi gayi (círculo lleno) con los resultados empíricos obtenidos por otros autores en $M$. hubbsi (círculo vacío).

Figure 8. Trend comparison between the theoretical result in Merluccius gayi gayi (filled circle) and the empirical result obtained by other authors in $M$. hubbsi (empty circle). 
rra de $47 \mathrm{~mm}$, sólo se estaría capturando el 0,23\% de los individuos inferiores a esta longitud total, se llega a la inevitable conclusión que un dispositivo con $d=47 \mathrm{~mm}$ permitiría que casi el $100 \%$ de los individuos vulnerables por el arte de pesca, y que por primera vez van a reproducirse (es decir, el 50\% está maduro sexualmente), alcancen a hacerlo antes de ser capturados. En consecuencia, se fortalecería el potencial reproductivo de la especie. Esto en cierta medida aseguraría reclutamientos exitosos, los que debieran mantener, al menos, un umbral inferior de biomasa estable.

No obstante lo anterior, y dado que los estudios de selectividad se orientan a conservar el potencial reproductivo de la especie (Pavez, 1989), resulta necesario establecer la separación inter-barra con datos diferenciados por sexo y, particularmente, con aquellos colectados durante los principales períodos de desove de la especie (agosto-noviembre), dado que esta condición podría afectar la selectividad del sistema. Una vez fijada así la separación entre barras e implementado el sistema en la flota arrastrera, podría ser aceptable la continuidad operacional de las embarcaciones durante todo el año y en cualquier zona, como ha resultado ser en otras pesquerías del mundo en que se ha implementado algún sistema de rejilla.

\section{CONCLUSIONES}

El análisis de los antecedentes recopilados, en relación a sistemas de grillas selectivas, sugiere que estos dispositivos podrían ser aplicados exitosamente en la pesquería industrial de arrastre de merluza común. Por otro lado, al comparar los resultados teóricos logrados en este estudio con los resultados obtenidos de experiencias con rejillas en merluza argentina, es posible indicar por analogía que el método propuesto se ajusta a lo observado en la realidad, por lo que representa una herramienta de análisis en el estudio de selectividad de dispositivos de rejilla.

Teniendo en consideración la talla de primera madurez sexual de la merluza común, se determina que la separación inter-barra adecuada de la rejilla debiera ser de $47 \mathrm{~mm}$. Con esto se lograría una longitud de captura $\left(l_{50}\right)$ de $390 \mathrm{~mm}$, una longitud de reclutamiento al arte $\left(l_{25}\right)$ de $381 \mathrm{~mm}$ y un estrecho rango de selección $(R S=18,25 \mathrm{~mm})$.
Estudios posteriores en esta línea debieran orientarse a validar el método, mediante pruebas de terreno en merluza común. Adicionalmente, debiera considerarse en el análisis la diferenciación de sexos. Finalmente, el mejoramiento del método planteado debiera considerar aspectos tales como el grado de contracción del pez y el flujo de agua a través de las rejillas, como los más relevantes.

\section{AGRADECIMIENTOS}

El autor agradece la colaboración brindada por la empresa pesquera El Golfo S.A. y Bío Bío S.A., en particular a los Sres. Ludwig Ziller y Patricio Herrera (Jefes de Flota), por facilitar las condiciones para efectuar la colecta de registros abordo. Asimismo, se agradece al Dr. Aquiles Sepúlveda, del Instituto de Investigación Pesquera, VIII Región, por sus invaluables comentarios y sugerencias en la elaboración del documento.

\section{REFERENCIAS}

Alarcón, R. y H. Arancibia. 1993. Talla de primera madurez sexual y fecundidad parcial en la merluza común, Merluccius gayi gayi (Guichenot, 1848). Cienc. y Tec. Mar, CONA 16: 33-45.

Anónimo. 1996. Sort- $X$ - News. (Boletín Informativo) 2/96, Selfi A.S., Troms $\varnothing$, Norway, August 1996.

Anónimo. 1998. Sort-X Selectivity (Fishing Technology) Manual. Hafi Ltd. F y Illingsdalen, Norway, $25 \mathrm{pp}$.

Arana, P. 1970. Estudio sobre la selectividad de la merluza (Merluccius gayi gayi $\mathrm{G}$.) por las mallas de los artes de arrastre en la zona de Valparaíso. Inv. Mar., 1(1): 39 pp.

Efron, B. 1981. Nonparametric estimates of standard error: the jackknife, the bootstrap and other methods. Biometrika, 68(3): 589-599.

Efron, B. 1985. Bootstrap confidence intervals for a class of parametric problems. Biometrika, 72(1): 45-58. 
Ercoli, R., J. García, A. Aubone, L. Salvini y R. Bertolo. 1998. Selectivity experiences on hake (Merluccius hubbsi) with different inter rod distances in a single-grid sorting device (DEJUPA) and the use of a special grid retention codend design. CM 1998/OPEN: 9. Fish Capture Committee. International Council for the Exploration of the Sea (ICES).

Ercoli, R., L. Salvini, A. Izzo J. García y J. Bartozzetti. 1997. Selectivity experiences on hake (Merluccius hubbsi) by means the use of single grid sorting device for the escape of juvenile fishes in trawls (DEJUPA). Poster CM 1997/HH:23 Annual Science Conference. International Council for the Exploration of the Sea (ICES). Baltimore, USA, 1997.

Fryer, R. y J. Sheperd. 1993. Models of codend selection. Gear selectivity/Technical interactions Symposium. NAFO SCR Doc. 93/123.

Gálvez, M. 1998. Distribución y abundancia de merluza común (Merluccius gayi) entre la V y VIII Regiones, durante 1997. In: Segundo Informe de Avance, FIP Nº6-33: 5-76.

Gálvez, M., H. Rebolledo, Ch. Cuevas, S. Lillo y M. Espejo. 1999. Análisis de selectividad en la pesquería de merluza común en la zona centro-sur. Informe de Avance, Proyecto FIP Nº6-25, 33 pp.

Gálvez, M., A. Sepúlveda, Ch. Díaz, L. Miranda, H. Rebolledo y Ch. Cuevas. 1998. Biomasa estacional de merluza común por área barrida por la flota industrial en la zona centro-sur. Pre Informe Final, FIP Nº96-33, 182 pp.

García, S.M. 1996. The precautionary approach to fisheries and its implications for fishery research, technology and management: an updated review. In: Precautionary approach to fisheries. FAO Fish. Tech. Paper, 350 (Part 2): 1-75.

Isaksen, B. 1996. Grids in Danish seines. Draft: Reporter's notes. Study group on grid (grate) sorting systems in trawls, beam trawls, and seine nets. Woods Hole, Massachusetts, USA, 13 \& 14 April 1996.

Isaksen, B. 1998a. Ensayos en Namibia con mallas cuadradas. Revista Redes de la Industria Pesquera, 102: 38

Isaksen, B. 1998b. Selectividad - La experiencia noruega. Revista Redes de la Industria Pesquera, 103:38-46.
Instituto de Investigación Pesquera (INPESCA). 1996. Análisis de la pesquería de merluza común (Merluccius gayi), evaluación del stock y estimación de la captura total permisible 1997. Inf. Tec., 14 pp.

Klenner, R.A. 1978. Selectividad de copo cubierto en red de arrastre de merluza en la zona de Valparaíso. Tesis, Esc. Cs. Mar y Alim. Univ. Católica de Valparaíso, 95 pp.

Larsen, R. 1996. The size-selective Sort-X grid system: construction, selectivity trials, and practical use. Draft: Reporter's notes. Study group on grid (grate) sorting systems in trawls, beam trawls, and seine nets. Woods Hole, Massachusetts, USA, 13 and 14 April 1996.

Larsen, R.B. y B. Isaksen. 1993. Size selectivity of rigid sorting grid in bottom trawls for Atlantic cod (Gadus morhua) and haddock (Melanogrammus aeglefinus). ICES Mar. Sci. Symp., 196: 178-182.

Millar, R.B. 1993. Incorporation between-haul variation using bootstrapping and nonparametric estimation of selection curves. Fish. Bull., 91:564572 .

Millar, R.B. y S.J. Walsh. 1992. Analysis of trawl selectivity studies with an application to trouser trawls. Fish. Res., 13: 205-220.

Omoto, S., T. Tokai y M. Tanda. 1998. Comparison of selectivity curve between square-mesh and diamond-mesh codends by AIC. Nippon Suisan Gakaishi, 64(3): 447-452. (en japonés).

Pavez, P. 1981. Determinación del esquema de selectividad de merluza común (Merluccius gayi gayi) por mallas de redes de arrastre de seis paneles. In: Perspectivas de Desarrollo de las Pesquerías Nacionales. Evaluación de los Principales Recursos Demersales entre Papudo (32²0') y Chiloé (4200'). CORFO: AP82-9.

Pavez, P. 1989. Implicaciones de una medida de regulación. Situación del tamaño mínimo de malla en redes de arrastre en la pesquería de merluza común (Merluccius gayi Guichenot 1848). Rev. Pacífico Sur (Num. Especial), 717-726.

Payá, I., S. Mora, H. Pool, H. Miranda, J. Ortega y R. Saldías. 1995. Cuantificación del reclutamiento de merluza común en la zona centro-sur. Informe Final Proyecto FIP N94-21, 66 pp. + anexos. 
Pikitch, E.K., P. Suuronen, D. Erickson y J.A. PérezComas. 1995. Codend size-selection: Good concept, but does it really work? Solving Bycatch. Considerations for today and tomorrow. Alaska Sea Grant College Program, Report N96-03, University of Alaska, Fairbanks: 107-114.

Pope, J.A., A.R. Margetts, J.M. Hamley y E.F. Akyuz. 1975. Manual of methods for fish stock assessment. Part III. Selectivity of fishing gear. FAO Fish. Tech. Paper, 41: 65 pp.
Saetersdal, G. y L. Villegas. 1968. Informe sobre experimentos de selectividad con red de arrastre. Boletín Científico $\mathrm{N}^{\circ} 9$. Instituto de Fomento Pesquero, 16 pp.

Suuronen, P. 1995. Conservation of young fish by management of trawl selectivity. Finnish Fish. Res., 15: 97-116.

Tokai, T., S. Omoto, R. Sato y K. Matuda. 1996. A method of determining selectivity curve of separator grid. Fish. Res., 27: 51-60. 